\title{
Glycyrrhizic acid nanoparticles inhibit LPS-induced inflammatory mediators in 264.7 mouse macrophages compared with unprocessed glycyrrhizic acid
}

\author{
This article was published in the following Dove Press journal: \\ International Journal of Nanomedicine \\ II April 2013 \\ Number of times this article has been viewed
}

\author{
Wei Wang ${ }^{1-3}$ \\ Meng Luo ${ }^{1-3}$ \\ Yujie $\mathrm{Fu}^{1-3}$ \\ Song Wang ${ }^{1-3}$ \\ Thomas Efferth ${ }^{4}$ \\ Yuangang $\mathrm{Zu}^{1-3}$ \\ 'Key Laboratory of Forest Plant \\ Ecology, ${ }^{2}$ Engineering Research \\ Center of Forest Bio-Preparation, \\ ${ }^{3}$ State Engineering Laboratory of \\ Bio-Resource Eco-Utilization, \\ Northeast Forestry University, \\ Harbin, People's Republic of China \\ ${ }^{4}$ Department of Pharmaceutical \\ Biology, Institute of Pharmacy and \\ Biochemistry, University of Mainz, \\ Mainz, Germany
}

\begin{abstract}
Glycyrrhizic acid (GA), the main component of radix glycyrrhizae, has a variety of pharmacological activities. In the present study, suspensions of GA nanoparticles with the average particle size about $200 \mathrm{~nm}$ were prepared by a supercritical antisolvent (SAS) process. Comparative studies were undertaken using lipopolysaccardide(LPS)-stimulated mouse macrophages RAW 264.7 as in vitro inflammatory model. Several important inflammation mediators such as NO, PGE 2 , TNF- $\alpha$ and IL- 6 were examined. These markers were highly stimulated by LPS and were inhibited both by nano-GA and unprocessed GA in a dose-dependent manner, especially $\mathrm{PGE}_{2}$ and TNF- $\alpha$. However nano-GA and unprocessed GA inhibited NO only at a high concentration. In general, we found that GA nanoparticle suspensions exhibited much better anti-inflammatory activities compared to unprocessed GA.
\end{abstract}

Keywords: glycyrrhizic acid, nanoparticle, mouse macrophages RAW 264.7, inflammatory cytokines

\section{Introduction}

Inflammation is a natural biological response to injury or infection in the human body. Various factors, such as microbial infections, chemicals, and immunologic reactions can cause inflammation. ${ }^{1}$ Prolonged inflammation can be harmful, contributing to the pathogenesis of many diseases, including rheumatoid arthritis, obesity, cardiovascular diseases, neurodegenerative diseases, diabetes, and cancer. ${ }^{2,3}$

Macrophages are considered to play essential roles in inflammation. If activated by endotoxins, macrophages produce inflammatory cytokines, which in turn activate other macrophages and other nearby cells to promote, for example, inducible nitric oxide (NO) synthase gene expression. ${ }^{4}$ Much progress has been made in the delineation of cell signaling pathways, in which the inflammation initiates a cascade of events that result in the overproduction of certain inflammation-associated genes and proinflammatory cytokines. ${ }^{5}$

Inhibition of inflammatory cytokine and mediator production serves as a key mechanism in the control of inflammation. A number of anti-inflammatory molecules have already entered clinical trials for the treatment of inflammatory disorder such as interleukin-1 (IL-1), interleukin-6 (IL-6), tumor necrosis factor- $\alpha$ (TNF- $\alpha$ ), prostaglandin $\mathrm{E}_{2}\left(\mathrm{PGE}_{2}\right)$ or nitric oxide (NO). ${ }^{6}$ Drugs that suppress the expression of these inflammatory mediators have therefore attracted significant interest as potential therapeutics for the treatment of inflammatory diseases. ${ }^{3}$
Correspondence: Yuangang Zu

Tel +8645182191314

Fax +86 45। 8210 2082

Email zygorl@vip.hl.cn 
Glycyrrhizic acid (GA), a terpenoid compound, is the main component of radix glycyrrhizae. GA has a variety of pharmacological activities such as antioxidative, anti-inflammatory, antiulcerous, antidotal, antiallergic, antiviral, immunomodulating, hepatoprotective, and cardioprotective properties. ${ }^{7-10}$ However, GA is slowly absorbed in vivo because of its poor water solubility. Large number of studies have shown that the reduction of the drug particle size to the nanometer scale considerably increases the activity and bioavailability of the drug. ${ }^{11}$ Supercritical antisolvent (SAS) process is an environmentally friendly technology developed in recent years. In this process, the drug is firstly dissolved in the solvent and then the drug solution is quickly sprayed into supercritical fluids (the antisolvent). Precipitation occurs immediately by a rapid recrystallization of the drug. In general, the SAS process can control the production of particle sizes within the nanometer range, and the solvent can be fully recovered. It is suitable to prepare nanocrystals of drugs or biologicaly active substance because of low temperature and inertia.

This study explored the anti-inflammatory activity and mechanisms of GA nanoparticles prepared by SAS in lipopolysaccharide (LPS)-stimulated RAW 264.7 macrophages compared to unprocessed GA particles.

\section{Materials and methods Materials}

GA ( $\geq 98 \%$ ) for nanosuspension preparation was purchased from Xi'an Green-Life Natural Products Co, Ltd (Xi'an, People's Republic of China). GA (99\%) for quantitative analysis was purchased from Sigma-Aldrich (St Louis, MO, USA). Compound glycyrrhizin tablets were manufactured by Minophagen Pharmaceutical Co., Ltd (Tokyo, Japan). LPS was purchased from Sigma-Aldrich. Griess reagent kit was obtained from Biyuntian Biological Technology Co., Ltd (Shanghai, People's Republic of China). PGE 2 , TNF- $\alpha$, and IL-6 enzyme-linked immunosorbent assay kits were purchased from 4A Biotech Co., Ltd (Beijing, People's Republic of China). 3-(4,5-dimethylthiazol-2-yl)-2,5-diphenyltetrazolium bromide (MTT) was purchased from Sigma-Aldrich. High-performance liquid chromatography grade methanol was purchased from J\&K Chemical Ltd (Beijing, People's Republic of China). Purified water was obtained from a Milli-Q ${ }^{\circledR}$ system from EMD Millipore (Billerica, MA, USA). All other chemicals were of analytical reagent grade.

\section{Preparation of GA nanosuspension}

Two processes were performed to obtain the final GA nanoparticle suspension. First, the micronized GA was prepared with an SAS apparatus modified from a carbon dioxide supercritical extraction apparatus by using dimethyl sulfoxide (DMSO) as the solvent and carbon dioxide as the antisolvent. Under the optimum conditions, micronized GA with a particle size about $200 \mathrm{~nm}$ was obtained.

\section{Surface morphology determined by scanning electron microscopy (SEM) of nanoparticles and unprocessed GA}

The samples were fixed to an SEM stub and sputter coated with gold using SBC-12 ion sputter coater (KYKY Technology Development Ltd, Beijing, People's Republic of China) to form a carbon conductive film. The surface morphology of the particles was then observed by SEM (Quanta $^{\text {TM }}$ 200; FEI Company, Hillsboro, OR, USA).

\section{Cell culture}

The RAW 264.7 cells, a murine macrophage-like cell line, were obtained from China Cell Line Bank (Beijing, People's Republic of China). RAW 264.7 cells were cultured in Dulbecco's modified Eagle's medium supplemented with $10 \%$ fetal bovine serum, $2 \mathrm{mM}$ glutamine, $100 \mathrm{U} / \mathrm{mL}$ penicillin, and $100 \mu \mathrm{g} / \mathrm{mL}$ streptomycin. Cells were incubated at $37^{\circ} \mathrm{C}$ in a humidified atmosphere ( $5 \%$ carbon dioxide). The test compounds were diluted with 2\% Dulbecco's modified Eagle's medium to the appropriate concentrations and added 1 hour before LPS treatment.

\section{MTT assay for cell viability}

The MTT assay was performed to measure cell viability. RAW 264.7 cells were mechanically scraped, seeded in 96-well plates, and incubated in a $37^{\circ} \mathrm{C}, 5 \%$ carbon dioxide incubator overnight. After 24 hours, cells were treated with different concentrations of GA nanoparticles and unprocessed GA or glycyrrhizin tablets for 24 hours. Subsequently, $20 \mu \mathrm{L}$ of $5 \mathrm{mg} / \mathrm{mL}$ MTT in fetal bovine serum-free medium was added to each well, and the cells were incubated for 4 hours. MTT was removed and resolved with $150 \mu \mathrm{L} /$ well dimethyl sulfoxide. The optical density was measured at $492 \mathrm{~nm}$ using a microplate reader (Tecan Infinite ${ }^{\circledR}$ M200, Tecan Group Ltd, Männedorf, Austria). Concentrations were determined for three wells of each sample, and this experiment was done in triplicate.

\section{Analysis of $\mathrm{NO}$ production}

Nitrite, a stable product of NO, was used to assess NO production based on the Griess reaction. RAW 264.7 cells were incubated with different concentrations of GA nanoparticles and unprocessed GA or glycyrrhizin tablets in the absence or presence of $1 \mu \mathrm{g} / \mathrm{mL}$ LPS. After 24 hours of incubation, the level of NO production was monitored by measuring the 
nitrite concentration in the supernatant of cultured medium using the Griess reagent assay. For the NO assay, $50 \mu \mathrm{L}$ supernatant of cultured medium was mixed with the same volume of Griess reagent. The absorbance was measured at $540 \mathrm{~nm}$ on a microplate reader. The concentration of NO in the media of sample-treated cells was calculated using the standard curve obtained for sodium nitrite dissolved in Dulbecco's modified Eagle's medium. Concentrations were determined for three wells in each sample, and this experiment was done in triplicate.

\section{Determination of PGE, TNF- $\alpha$, and IL-6 levels}

To investigate the effect of GA nanoparticles and unprocessed GA or glycyrrhizin tablets on cytokine responses from LPS-treated cells, RAW 264.7 cells seeded on 96-well plates were treated with 303, 606, and $909 \mu \mathrm{M}$ GA 1 hour before treatment with $1 \mu \mathrm{g} / \mathrm{mL}$ LPS for 24 hours in a $37^{\circ} \mathrm{C}$, $5 \%$ carbon dioxide incubator. Cell-free supernatants were collected and stored at $-20^{\circ} \mathrm{C}$ until assayed for cytokines. The concentrations of $\mathrm{PGE}_{2}, \mathrm{TNF}-\alpha$, and IL-6 in the culture supernatants of RAW 264.7 cell cultures were determined using an enzyme-linked immunosorbent assay kit. Concentrations were determined for three wells in each sample, and this experiment was done in triplicate.

\section{Statistical analysis}

The experimental values were represented as arithmetic mean \pm standard deviation. The unpaired Student's $t$-test was used to determine the statistical significance. Statistically, a $P$-value less than 0.05 was considered to be significant and a $P$-value less than 0.01 was considered to be very significant.

\section{Results}

\section{Surface morphology of GA nanoparticles and unprocessed GA}

Figure 1A and B show the particle size of unprocessed GA material and GA nanoparticle suspensions performed by scanning electron microscopy (SEM). The final GA nanosuspension prepared by SAS contained particles of about $200 \mathrm{~nm}$, whereas GA raw materials exhibited an irregular shape and micrograde particle size.

\section{Cell viability and comparative effects of GA nanoparticles and unprocessed GA on LPS-induced NO production}

Unprocessed GA, nano-GA and glycyrrhizin tablets up to $909 \mu \mathrm{M}$ did not show cytotoxic effects (data not shown).
Nitric oxide (NO) is a short-living free radical that is produced from L-arginine by catalytic reactions of NO synthases in mammalian immune, cardiovascular and neural systems, where it functions as signaling molecule. ${ }^{12}$ To analyze potential anti-inflammatory properties of GA nanoparticles and unprocessed GA, we used murine RAW 264.7 macrophage cells, which produce NO upon stimulation with LPS. The amount of produced NO was determined by measurement of nitrite, a stable metabolite of NO. Cells were pretreated with GA nanoparticles or unprocessed GA, and then stimulated with $1 \mu \mathrm{g} / \mathrm{mL}$ LPS. The control group was untreated with both LPS and samples. After the cell culture media were collected, nitrite levels were determined. During the incubation time of 24 hours, RAW 264.7 macrophages produced $2.68 \pm 0.34 \mu \mathrm{M}$ nitrite in the resting state, whereas after LPS $(1 \mu \mathrm{g} / \mathrm{mL})$ stimulation, NO production dramatically increased to $20.12 \pm 3.69 \mu \mathrm{M}$. Nano-GA and unprocessed GA inhibited nitrite production 24 hours after LPS stimulation in a dose-dependent manner (Figure 2). At 303 $\mu \mathrm{M}$, nano-GA and unprocessed $\mathrm{GA}$ reduced NO production by $13.33 \%$ and $0.03 \%$, respectively. At concentrations of $606 \mu \mathrm{M}$, nano-GA and unprocessed GA reduced NO production by $51.44 \%$ and $18.67 \%$, respectively, whereas at 909 $\mu \mathrm{M}$, nano-GA and unprocessed GA reduced NO production by $87.78 \%$ and $23.56 \%$, respectively. Nano-GA inhibited NO generation much better than unprocessed GA, especially at high concentrations. When compared to the positive control (glycyrrhizin tablets), the inhibition rate of NO was: nano-GA $>$ positive control $>$ unprocessed GA.

\section{Comparative effects of GA nanoparticles and unprocessed GA on LPS-induced $\mathrm{PGE}_{2}$ production}

Levels of the pro-inflammatory lipid mediator $\mathrm{PGE}_{2}$ were analyzed upon LPS stimulation in RAW 264.7 cells. During the incubation for 24 hours, RAW 264.7 macrophages produced $19.75 \pm 5.24 \mathrm{pg} / \mathrm{mL} \mathrm{PGE}_{2}$ in the resting state, whereas after LPS stimulation, $\mathrm{PEG}_{2}$ production dramatically increased to $628.47 \pm 33.59 \mathrm{pg} / \mathrm{mL}$. Both GA nanoparticles and unprocessed $\mathrm{GA}$ reduced $\mathrm{PEG}_{2}$ production in a dose-dependent manner (Figure 3). At concentrations of $303 \mu \mathrm{M}$, GA nanoparticles and unprocessed GA reduced $\mathrm{PGE}_{2}$ production by $59.38 \%$ and $27.18 \%$, respectively. Nano-GA exhibiting more than two-fold inhibition activity than unprocessed GA. At $606 \mu \mathrm{M}, \mathrm{PGE}_{2}$ production was reduced by $73.22 \%$ and $47.49 \%$, respectively, whereas at $909 \mu \mathrm{M}$, nano-GA and unprocessed GA reduced $\mathrm{PGE}_{2}$ production by $86.78 \%$ and $66.90 \%$, respectively. Nano-GA inhibited $\mathrm{PGE}_{2}$ generation much better than unprocessed 

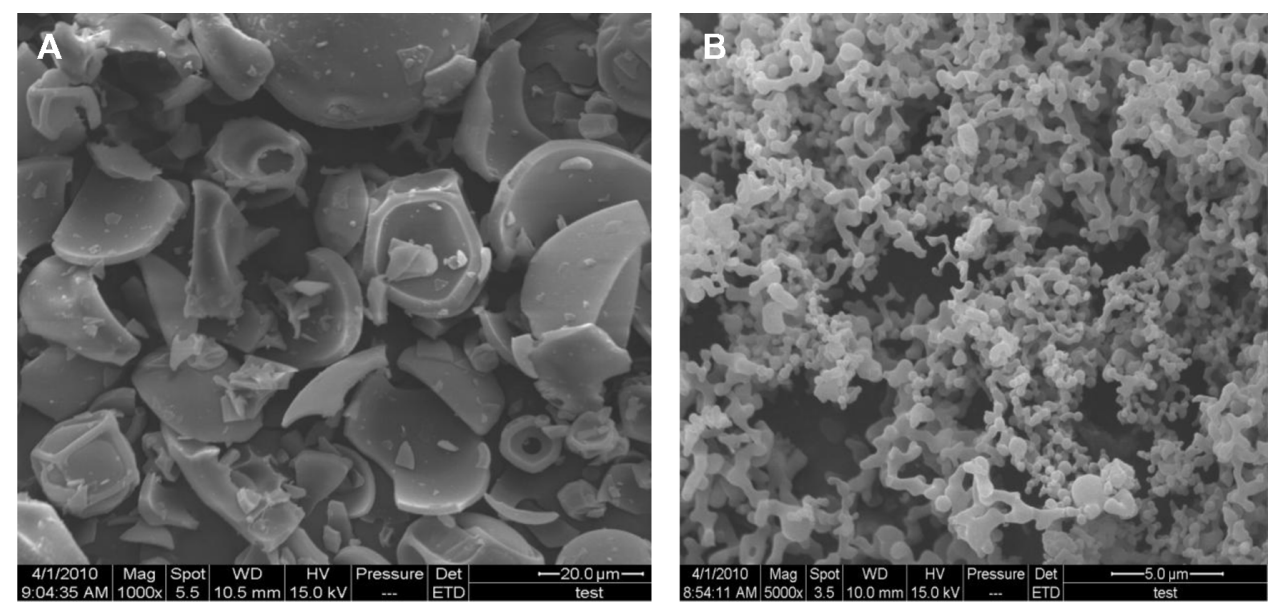

Figure I Scanning electron microscopy result of (A) unprocessed GA and (B) GA nanoparticles. Abbreviation: GA, glycyrrhizic acid.

GA. When compared to the positive control (glycyrrhizin tablets), the inhibition rate of $\mathrm{PGE}_{2}$ was: nano-GA $>$ positive control $>$ unprocessed GA.

\section{Comparative effects of GA nanoparticles and unprocessed GA on LPS-induced TNF- $\alpha$ production}

TNF- $\alpha$ is one of the most important cytokines and is required for the induction of NO synthesis in LPS-stimulated macrophages. TNF- $\alpha$ elicits a number of physiological effects, such as septic shock, inflammation, cachexia, and cytotoxicity. ${ }^{13}$ TNF- $\alpha$ concentrations in the culture supernatants of RAW 264.7 cells were measured by enzyme-linked immunosorbent assay (Figure 4). RAW 264.7 cells treated

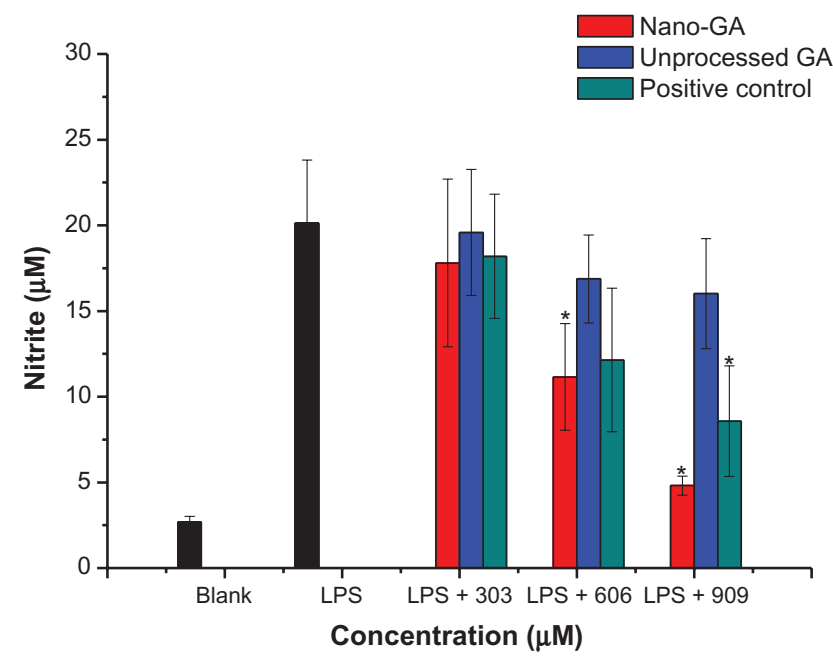

Figure 2 Effect of different concentrations of GA nanoparticles and unprocessed GA on LPS-induced nitric oxide.

Note: $* P<0.05$, significantly different from the LPS-treated group by unpaired Student's t-test.

Abbreviations: GA, glycyrrhizic acid; LPS, lipopolysaccharide. with LPS produced significant amounts of TNF- $\alpha$. The concentration of TNF- $\alpha$ increased from $1.47 \pm 0.27 \mathrm{ng} /$ $\mathrm{mL}$ to $62.99 \pm 9.56 \mathrm{ng} / \mathrm{mL}$ after LPS stimulation. However, the concentrations of TNF- $\alpha$ in the supernatant of cells treated with GA nanoparticles and unprocessed GA were significantly decreased compared to the LPS control group. At concentrations of $303 \mu \mathrm{M}$, GA nanoparticles and unprocessed GA reduced TNF- $\alpha$ production by $76.94 \%$ and $57.01 \%$, respectively. At $606 \mu \mathrm{M}$, TNF- $\alpha$ production was reduced by $79.61 \%$ and $69.02 \%$, respectively, whereas at $909 \mu \mathrm{M}$, TNF- $\alpha$ production was reduced by $89.51 \%$ and $69.58 \%$, respectively. Both nano-GA and unprocessed GA strongly inhibited TNF- $\alpha$ generation. When compared to the positive control (glycyrrhizin tablets), the reduction

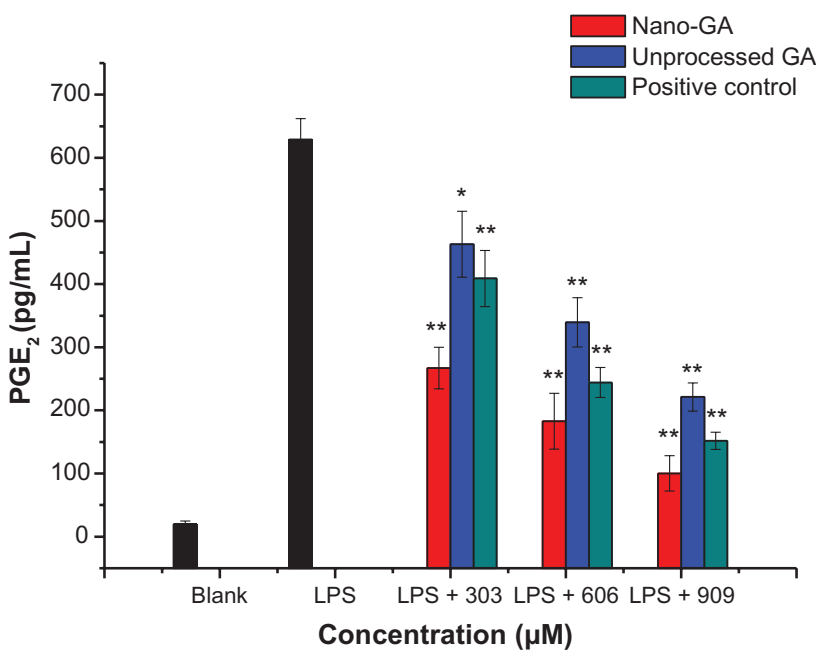

Figure 3 Effect of different concentrations of GA nanoparticles and unprocessed GA on LPS-induced PGE 2 .

Notes: $* P<0.05$; $* * P<0.0$ I, significantly different from the LPS-treated group by unpaired Student's $t$-test.

Abbreviations: GA, glycyrrhizic acid; LPS, lipopolysaccharide; $P E_{2}$, prostaglandin $E_{2}$. 


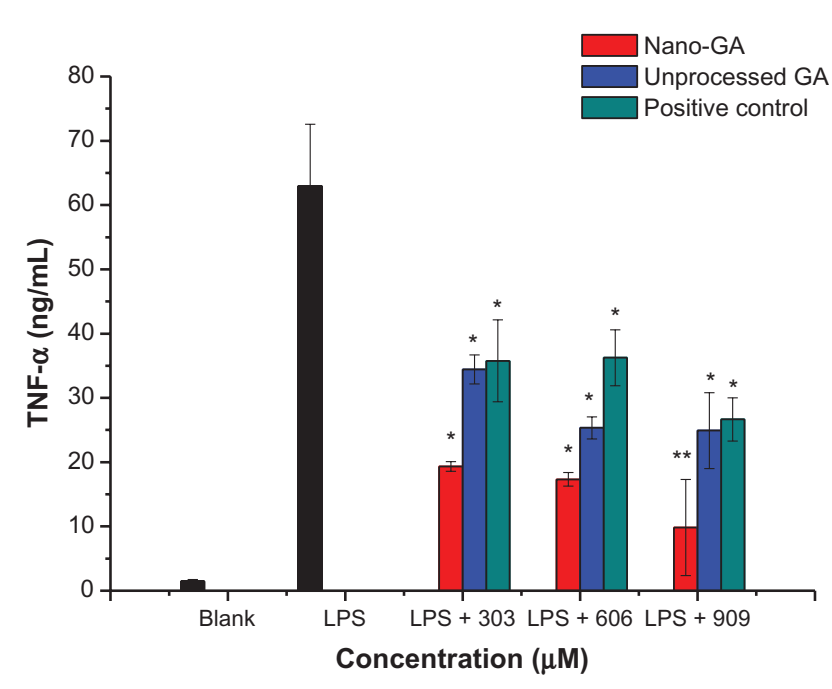

Figure 4 Effect of different concentrations of GA nanoparticles and unprocessed GA on LPS-induced TNF- $\alpha$.

Notes: $* P<0.05 ; * * P<0.01$, significantly different from the LPS-treated group by unpaired Student's $t$-test.

Abbreviations: GA, glycyrrhizic acid; LPS, lipopolysaccharide; TNF- $\alpha$, tumor necrosis factor- $\alpha$.

rate of TNF- $\alpha$ was: nano-GA $>$ unprocessed GA $>$ positive control.

\section{Comparative effects of GA nanoparticles and unprocessed GA on LPS-induced IL-6 production}

IL-6 represents an endogenous mediator of LPS-induced fever. RAW 264.7 cells treated with LPS produced significant amounts of IL-6, increasing from $11.67 \pm 3.06 \mathrm{pg} / \mathrm{mL}$ to $320.33 \pm 11.59 \mathrm{pg} / \mathrm{mL}$ after LPS stimulation (Figure 5). IL-6 in the supernatant of cells treated with GA nanoparticles and unprocessed GA did not significantly change compared to the LPS control group. At concentrations of $303 \mu \mathrm{M}$, GA nanoparticles and unprocessed GA reduced IL-6 production by $44.39 \%$ and $20.79 \%$, respectively. At $606 \mu \mathrm{M}$, IL-6 production was reduced by $48.85 \%$ and $23.92 \%$, respectively, whereas at $909 \mu \mathrm{M}$, IL-6 production was reduced by $57.67 \%$ and $36.66 \%$, respectively. When compared to the positive control (glycyrrhizin tablets), the reduction rate of IL- 6 was: nano-GA $>$ positive control $>$ unprocessed GA. Nano-GA inhibited IL-6 generation much better than unprocessed GA.

\section{Discussion}

Inflammation is the primary response of the immune system against infection or irritation, and macrophages play a crucial role during the inflammatory process. ${ }^{14}$ In the presence of stimuli such as LPS, macrophages are activated and produce

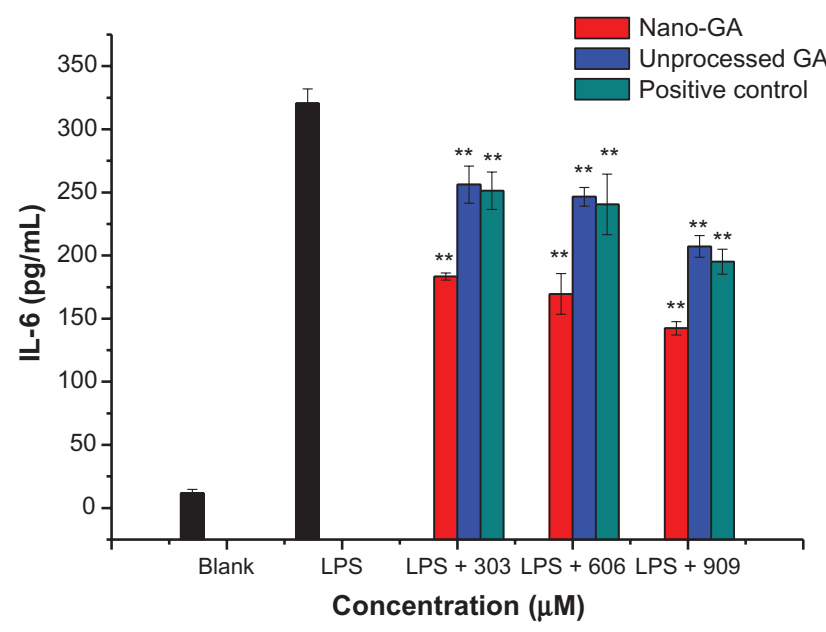

Figure 5 Effect of different concentrations of GA nanoparticles and unprocessed GA on LPS-induced IL-6.

Note: $* * P<0.01$, significantly different from the LPS-treated group by unpaired Student's t-test.

Abbreviations: GA, glycyrrhizic acid; LPS, lipopolysaccharide;IL-6, interleukin-6.

various cytokines such as TNF- $\alpha$, IL-1 $\beta$, IL-6, and IL-10, as well as inflammatory mediators such as $\mathrm{NO}$ and $\mathrm{PGE}_{2} \cdot 3,15,16$ Therefore, LPS-activated macrophages have typically been used to evaluate the anti-inflammatory effects of various materials. The production of these cytokines and mediators may result in the systemic inflammatory response syndrome, severe tissue damage, and septic shock. ${ }^{17}$ Therefore, agents that regulate cytokines and inflammatory mediators may have therapeutic effects.

Glycyrrhiza uralensis (Leguminosae) has long been used throughout the world as a sweetener and in folk medicine because of its antioxidative, anti-inflammatory, antibacterial, antiangiogenic, and antiallergenic properties. ${ }^{1}$ Its main components are considered to be the triterpene saponins glycyrrhizin and GA. ${ }^{18-20}$

Nanomaterials are important materials due to their unique physical and chemical properties. ${ }^{21}$ Due to accumulation of nanoparticles in the cell by enhanced permeability and retention effect, nanoparticles achieve new biological properties. ${ }^{22,23}$ In the current report, solid dispersion microparticles of poorly water-soluble GA were prepared by using SAS method. This technique is a feasible and efficient way to enhance the solubility of poorly water-soluble GA with high dissolution rate.

In order to compare the anti-inflammatory activity of GA nanoparticles and unprocessed GA in LPS-stimulated RAW 264.7 macrophages, several important inflammation mediators were examined. We found that the release of $\mathrm{NO}, \mathrm{PGE}_{2}$, TNF- $\alpha$ and IL-6 release, which was highly stimulated by LPS, was inhibited by both nano-GA and unprocessed GA 
in a dose-dependent manner. In particular, GA suspensions suspension, compared with the unprocessed GA significantly enhanced the reduction of $\mathrm{PGE}_{2}$ and TNF- $\alpha$ even at low concentrations, but did not significantly affect NO production at low concentrations. Nano-GA exhibited much better inhibition activities compared to unprocessed GA. This is due to the fact that nanoparticles have a smaller particle sizes compared to unprocessed drug particles, which leads to an increase of the interfacial surface area and consequently improvement of water solubility, allowing for smaller dosages and more rapid and direct usage of otherwise poorly water-soluble drugs. ${ }^{24}$ When compared to the positive control (glycyrrhizin tablets) the reduction rate of $\mathrm{NO}, \mathrm{PGE}_{2}$, and $\mathrm{IL}-6$ was: nano-GA $>$ positive control $>$ unprocessed GA, whereas for TNF- $\alpha$ it was: GA nanoparticles $>$ unprocessed GA $>$ positive control.

\section{Conclusion}

In summary, the findings presented here suggest that GA nanoparticles prepared by the SAS method performed much better inhibition activity for LPS-induced NO, PGE 2 , TNF- $\alpha$, and IL-6 production in macrophage cells than unprocessed GA. Thus, GA nanoparticles may have therapeutic potential for the modulation and regulation of macrophage activation, and may provide safe and effective treatment options for a variety of inflammation-mediated diseases.

\section{Acknowledgements}

The authors gratefully acknowledge the financial supports by special scientific fund for non-profit public industry (201204601) and postdoctoral science-research developmental foundation of Heilongjiang province (LBH-Q11183).

\section{Disclosure}

The authors report no conflicts of interest in this work.

\section{References}

1. Shin EM, Zhou HY, Guo LY, et al. Anti-inflammatory effects of glycyrol isolated from Glycyrrhiza uralensis in LPS-stimulated RAW264.7 macrophages. Int Immunopharmacol. 2008;8(11): 1524-1532.

2. Laskin DL, Pendino KJ. Macrophages and inflammatory mediators in tissue injury. Аnпu Rev Pharmacol Toxicol. 1995;35:655-677.

3. Park YD, Jin CH, Choi DS, Byun MW, Jeong IY. Biological evaluation of isoegomaketone isolated from Perilla frutescens and its synthetic derivatives as anti-inflammatory agents. Arch Pharm Res. 2011;34(8):1277-1282.

4. Nathan C. Nitric oxide as a secretory product of mammalian cells. FASEB J. 1992;6(12):3051-3064.

5. O'Neill LA. Targeting signal transduction as a strategy to treat inflammatory diseases. Nat Rev Drug Discov. 2006;5(7):549-563.

6. Reinhart K, Karzai W. Anti-tumor necrosis factor therapy in sepsis: update on clinical trials and lessons learned. Crit Care Med. 2001; 29(Suppl 7):S121-S125.
7. Isbrucker RA, Burdock GA. Risk and safety assessment on the consumption of Licorice root (Glycyrrhiza sp.), its extract and powder as a food ingredient, with emphasis on the pharmacology and toxicology of glycyrrhizin. Regul Toxicol Pharmacol. 2006;46(3): 167-192.

8. Hennell JR, Lee S, Khoo CS, Gray MJ, Bensoussan A. The determination of glycyrrhizic acid in Glycyrrhiza uralensis Fisch. ex DC. (Zhi Gan Cao) root and the dried aqueous extract by LC-DAD. J Pharm Biomed Anal. 2008;47(3):494-500.

9. Kimura M, Moro T, Motegi $\mathrm{H}$, et al. In vivo glycyrrhizin accelerates liver regeneration and rapidly lowers serum transaminase activities in $70 \%$ partially hepatectomized rats. Eur J Pharmacol. 2008;579(1-3): 357-364.

10. Kurisu S, Inoue I, Kawagoe T, et al. Clinical profile of patients with symptomatic glycyrrhizin-induced hypokalemia. J Am Geriatr Soc. 2008;56(8):1579-1581.

11. Zhang L, Wei W, Zu YG, Yang L, Zhao XH, Zu BS. Comparative study on effects of GA nanoparticles suspension and unprocessed GA solution on oral bioavailability, hepatic function and liver antioxidant enzymes activities in carbon tetrachloride-induced liver damage rats. Mater Sci Forum. 2011;694:423-429.

12. Nathan $\mathrm{C}, \mathrm{Xie} \mathrm{QW}$. Regulation of biosynthesis of nitric oxide. J Biol Chem. 1994;269(19):13725-13728.

13. Aggarwal BB, Natarajan K. Tumor necrosis factors: developments during the last decade. Eur Cytokine Netw. 1996;7(2):93-124.

14. Ialenti A, Moncada S, Di Rosa M. Modulation of adjuvant arthritis by endogenous nitric oxide. Br J Pharmacol. 1993;110(2):701-706.

15. Rhule A, Navarro S, Smith JR, Shepherd DM. Panax notoginseng attenuates LPS-induced pro-inflammatory mediators in RAW264.7 cells. J Ethnopharmacol. 2006;106(1):121-128.

16. Ci X, Ren R, Xu K, et al. Schisantherin A exhibits anti-inflammatory properties by down-regulating NF- $\mathrm{KB}$ and MAPK signaling pathways in lipopolysaccharide-treated RAW 264.7 cells. Inflammation. 2010;33(2):126-136.

17. Shimazu R, Akashi S, Ogata H, et al. MD-2, a molecule that confers lipopolysaccharide responsiveness on Toll-like receptor 4. J Exp Med. 1999;189(11):1777-1782.

18. Akamatsu H, Komura J,AsadaY, NiwaY. Mechanism of anti-inflammatory action of glycyrrhizin: effect on neutrophil functions including reactive oxygen species generation. Planta Med. 1991;57(2):119-121.

19. Matsui S, Matsumoto H, Sonoda Y, et al. Glycyrrhizin and related compounds down-regulate production of inflammatory chemokines IL-8 and eotaxin 1 in a human lung fibroblast cell line. Int Immunopharmacol. 2004;4(13):1633-1644.

20. Chin YW, Jung HA, Liu Y, et al. Anti-oxidant constituents of the roots and stolons of licorice (Glycyrrhiza glabra). J Agric Food Chem. 2007;55(12):4691-4697.

21. Venkatasubbu GD, Ramasamy S, Avadhani GS, Palanikumar L, Kumar J. Size-mediated cytotoxicity of nanocrystalline titanium dioxide, pure and zinc-doped hydroxyapatite nanoparticles in human hepatoma cells. J Nanopart Res. 2012;14:819-836.

22. Kasemets K, Ivask A, Dubourguier HC, Kahru A. Toxicity of nanoparticles of $\mathrm{ZnO}, \mathrm{CuO}$ and $\mathrm{TiO}_{2}$ to yeast Saccharomyces cerevisiae. Toxicol in Vitro. 2009;23(6):1116-1122.

23. Lee BS, Park K, Park S, et al. Tumor targeting efficiency of bare nanoparticles does not mean the efficacy of loaded anticancer drugs: importance of radionuclide imaging for optimization of highly selective tumor targeting polymeric nanoparticles with or without drug. J Control Release. 2010;147(2):253-260.

24. Sui X, Wei W, Yang L, et al. Preparation, characterization and in vivo assessment of the bioavailability of glycyrrhizic acid microparticles by supercritical anti-solvent process. Int J Pharm. 2012;423(2): 471-479. 
International Journal of Nanomedicine

Dovepress

\section{Publish your work in this journal}

The International Journal of Nanomedicine is an international, peerreviewed journal focusing on the application of nanotechnology in diagnostics, therapeutics, and drug delivery systems throughou the biomedical field. This journal is indexed on PubMed Central, MedLine, CAS, SciSearch ${ }^{\circledR}$, Current Contents ${ }^{\circledR} /$ Clinical Medicine,
Journal Citation Reports/Science Edition, EMBase, Scopus and the Elsevier Bibliographic databases. The manuscript management system is completely online and includes a very quick and fair peer-review system, which is all easy to use. Visit http://www.dovepress.com/ testimonials.php to read real quotes from published authors.

Submit your manuscript here: http://www.dovepress.com/international-journal-of-nanomedicine-journal 